\title{
ON THE ORIGIN OF THE ABUNDANCE ANOMALIES IN IK PEG
}

\author{
B. SMALLEY ${ }^{1}$, K. C. SMITH ${ }^{2}$, D. WONNACOTT ${ }^{3}$ \\ 1. Dept of Physics, Keele University, Staffordshire, ST5 5BG \\ 2. Dept of Physics 83 Astronomy, UCL, London, WC1E 6BT \\ 3. MSSL, Holmbury St. Mary, Dorking, Surrey, RH5 $6 \mathrm{NT}$
}

\begin{abstract}
IK Peg is a binary system comprising a pulsating A-type star in orbit with a massive white dwarf. A detailed abundance analysis of IK Peg A has been performed. It is found that the $\mathrm{Ca}$ and Sc abundances are approximately solar, and the Fe-group elements slightly enhanced. IK Peg is not a classical Am star but the results are not inconsistent with its spectroscopic classification as a marginal Am star. An excess of $\mathrm{Ba}$ and $\mathrm{Sr}$ are found. These anomalies could be explained by radiative diffusion processes operating in the atmosphere of IK Peg A, even though it is undergoing small-amplitude pulsations. Alternatively, since the companion is a massive white dwarf, these anomalies could be the result of mass transfer during the common envelope phase of the binary system's evolution.
\end{abstract}

\section{Introduction}

The sixth-magnitude A-type star IK Peg (HR 8210) has been the subject of much interest over the last few years. It is a well-known single-lined spectroscopic binary with a period of $21.9 \mathrm{~d}$, but only recently has the companion (IK Peg B) been positively identified as a massive $\left(1.15 \mathrm{M}_{\odot}\right.$ ) hot $(35000 \mathrm{~K})$ white dwarf (Wonnacott, Kellett \& Stickland 1993). The primary itself (IK Peg A) is by no means an uninteresting main-sequence A-type star. It is known to be undergoing small amplitude pulsations (Kurtz 1978; Wonnacott et al. 1994) and exhibits mild spectroscopic peculiarities similar to those associated with the metallic-lined A-type stars (Cowley et al. 1969).

Because the system is a post common envelope binary (Sarna et al. 1995), we have performed a detailed elemental abundance analysis, in order to see if we can find any anomalies that may be the result of mass transfer 
from the white dwarf progenitor. Here we present a summary of the results given by Smalley et al. (1996).

\section{Results}

The results of the detailed elemental abundance analysis by Smalley et al. (1996) are summarized below:

$\begin{array}{lclclclc}\mathrm{EL} & {[\mathrm{EL} / \mathrm{H}]} & \mathrm{EL} & {[\mathrm{EL} / \mathrm{H}]} & \mathrm{EL} & {[\mathrm{EL} / \mathrm{H}]} & \mathrm{EL} & {[\mathrm{EL} / \mathrm{H}]} \\ \mathrm{C} & -0.34 \pm 0.18 & \mathrm{Si} & +0.13 \pm 0.16 & \mathrm{~V} & +0.07 \pm 0.16 & \mathrm{Cu} & +0.05 \pm 0.21 \\ \mathrm{~N} & +0.03 \pm 0.19 & \mathrm{~S} & -0.01 \pm 0.15 & \mathrm{Cr} & +0.13 \pm 0.19 & \mathrm{Zn} & +0.08 \pm 0.17 \\ \mathrm{O} & -0.19 \pm 0.19 & \mathrm{~K} & -0.24 \pm 0.14 & \mathrm{Mn} & +0.05 \pm 0.18 & \mathrm{Sr} & +0.49 \pm 0.17 \\ \mathrm{Na} & +0.07 \pm 0.13 & \mathrm{Ca} & -0.11 \pm 0.25 & \mathrm{Fe} & +0.17 \pm 0.17 & \mathrm{Y} & +0.08 \pm 0.15 \\ \mathrm{Mg} & -0.13 \pm 0.23 & \mathrm{Sc} & -0.19 \pm 0.20 & \mathrm{Co} & +0.22 \pm 0.15 & \mathrm{Zr} & -0.07 \pm 0.18 \\ \mathrm{Al} & -0.17 \pm 0.17 & \mathrm{Ti} & +0.26 \pm 0.24 & \mathrm{Ni} & +0.29 \pm 0.19 & \mathrm{Ba} & +1.13 \pm 0.17\end{array}$

\section{Conclusion}

A detailed abundance analysis of the pulsating A-type star IK Peg A has revealed that the $\mathrm{Ca}$ and $\mathrm{Sc}$ abundances are approximately solar and the Fe-group elements slightly enhanced. This star is not a classical Am star, but the results are not inconsistent with its spectroscopic classification as a marginal Am star. Whether this marginal Am character is due to the effects of radiative diffusion is unclear, because there is the possibility that some, or all, of the abundance anomalies may be the result of mass transfer.

The obvious excess of $\mathrm{Ba}$ and $\mathrm{Sr}$ may be evidence for contamination by the white dwarf progenitor during the common envelope phase of the binary system evolution. Smalley et al. (1996) suggested that IK Peg A is a hot F str $\lambda 4077$ star. The F str $\lambda 4077$ stars are, however, a heterogeneous class, with the cooler members being related to Barium stars, while the hotter members resembling the Am or $\delta$ Del stars (North, Berthet \& Lanz 1994). Thus, it is unclear as to the origin of the anomalies in the hot $\mathrm{F} \operatorname{str} \lambda 4077$ stars, since their similarities to Am stars raises the possibility that the anomalies are due to radiative diffusion and not mass transfer. In fact, even if there were any anomalies due to mass transfer they may well have been masked by the effects of subsequent radiative diffusion (North et al. 1994).

\section{References}

Cowley A., Cowley C., Jaschek H., Jaschek C., 1969, AJ, 74, 375

Kurtz, D.W., 1978, Ap. J., 221, 869

North P., Berthet S., Lanz T., 1995, A\&A, 281, 775

Sarna M.J., Dhillon V.S., Marsh T.R., Marks P.B., 1995, MNRAS, 272, L41

Smalley B., Smith K.C., Wonnacott D., Allen C.S., 1996, MNRAS, 278, 688

Wonnacott D., Kellett B.J., Stickland D.J., 1993, MNRAS, 262, 277

Wonnacott D., Kellett B.J., Smalley B., Lloyd C., 1994, MNRAS, 267, 1045 